\title{
Recent Activities on SST-1 and ADITYA-U Tokamaks*)
}

\author{
Promod K. SHARMA ${ }^{1,2)}$, Yogesh M. JAIN ${ }^{1,2)}$, Kiran K. AMBULKAR ${ }^{1)}$, Pramod R. PARMAR ${ }^{1)}$, \\ Chetan G. VIRANI ${ }^{1)}$, Saifali DALAKOTI ${ }^{1)}$, Jagabandhu KUMAR ${ }^{1)}$, Arvind L. THAKUR ${ }^{1)}$, \\ Daniel RAJU ${ }^{1,2)}$, Joydeep GHOSH ${ }^{1,2)}$ and SST-1 and ADITYA-U Team ${ }^{1)}$ \\ 1) Institute for Plasma Research, Bhat, Gandhinagar - 382428, Gujarat, India \\ 2) Homi Bhabha National Institute, Mumbai, Maharashtra, India
}

(Received 12 January 2018 / Accepted 12 April 2018)

\begin{abstract}
The successful operation of superconducting tokamaks is very challenging because of limitations arising out of a complex geometry, configuration and construction. The slow penetration of loop voltage because a continuous plasma chamber requires low loop voltage plasma initiation. Apart from these operational challenges, SST1 also has technical issues related to cooling down of the superconducting poloidal field (PF) coils, for obtaining advanced plasma operation and control. Furthermore, limits are imposed on the loop voltage by magnet power supplies and PF coil insulation. To improve our understanding of these issues, operations in SST-1 have been attempted with low loop voltages and will be discussed in this paper.

It is envisaged that designing and carrying out experiments in SST1, to understand above issues, may be very complex, time consuming and may not be feasible due to complexities in SST1 machine operational scenario. Hence Aditya machine upgradation was initiated with the understanding that solution to some of these problems may be obtained through experiments specially designed for this purpose in ADITYA-U, in parallel. Thus some of these experiments are also being planned to carry out in tokamak ADITYA-U, in particular, non-inductive current drive using lower hybrid waves, employing passive active multi-junction (PAM) antenna. The performance of the lower hybrid waves launched by the grill antenna is presented and the need for PAM antenna is presented. The design of the PAM antenna for ADITYA-U machine is also discussed.
\end{abstract}

(C) 2018 The Japan Society of Plasma Science and Nuclear Fusion Research

Keywords: tokamak, SST1, ADITYA-Upgrade, lower hybrid current drive

DOI: $10.1585 /$ pfr. 13.3502100

\section{Introduction}

Low loop voltage plasma initiation is envisaged in SST1 tokamak like in all the other superconducting tokamaks [1-5]. Furthermore in SST1 limits are imposed on the loop voltage by magnet power supplies and PF coil insulation. To overcome these issues, breakdown in SST1 [2] is attempted using LHCD system [6, 7]. Earlier breakdown and start-up with LHCD has been demonstrated in the presence of finite loop voltage generated by vertical field [8] and with Ohmic field [9]. In this paper we report the breakdown of plasma in SST1 machine without any loop voltage. Also the technical issues related to cooling down of the superconducting poloidal field (PF) coils, for obtaining advanced plasma operation and control, necessitated experiments to identify and find solutions to the problems. The results obtained from these experiments are also presented and discussed in the paper. It is obvious that until the technical issues of SST1 machine is identified and resolved, another simpler device is required where SST1 relevant experiments may be carried out and well understood to have confidence in the operation of SST1 when

author's e-mail: pramod@ipr.res.in

*) This article is based on the invited presentation at the 26th International Toki Conference (ITC26). all its issues are resolved. Hence ADITYA machine upgradation was undertaken. It provided an opportunity to explore non-inductive current drive experiments employing passive-active-multijunction (PAM) antenna. The paper is organized as follows: Section-2 describes the breakdown studies with LHCD followed by cool down experiments on SST1 machine in section-3. The preparative activities for non-inductive current drive using lower hybrid waves for ADITYA-U with PAM antenna and its need is presented in section- 4 followed by the conclusion in the last section.

\section{Breakdown Studies in SST1}

The LHCD system is employed to carry out breakdown studies in SST1 machine so that low loop voltage start-up experiments may be carried out in future. The rf power is launched through grill antenna which consists of two rows, each having 32 elements of waveguide, as shown in Fig. 1. The SST1 LHCD system has a unique design feature, which enables launching of waves having composite spectra. This is achieved by phasing each of the 32 elements of the grill antenna, in the top and bottom rows independently, relative to adjacent elements, so as to launch waves having different characteristics from each of 


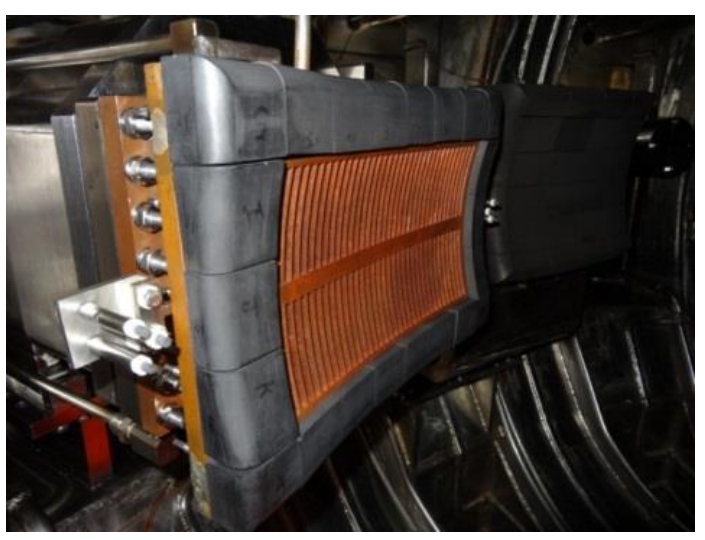

Fig. 1 Photograph of grill antenna which has two rows, each having 32 elements of waveguides.

the rows. Further each of the rows is fed by different sets of klystrons (each rated for $0.5 \mathrm{MW}$ continuous wave rf power at $3.7 \mathrm{GHz}$ ) which help in staggering the rf power from each row temporally.

In this configuration, one row of the grill (which consists of 32 elements of waveguide) is relatively phased (with respect to its adjacent waveguides) with zero degree $\left(\Delta \varphi=0^{\circ}\right)$, so that it can launch the waves in radial direction only, whereas the other row (which also has 32 elements) is relatively phased (with respect to its adjacent waveguides) with 130 degree $\left(\Delta \varphi=130^{\circ}\right)$ so as to launch travelling waves in toroidal direction. The cross section of the element is $76 \mathrm{~mm}$ (height) $\times 7 \mathrm{~mm}$ (width) and the elements are separated by a septum of $2 \mathrm{~mm}$. Hence the periodicity (center to center distance between adjacent elements) of the grill antenna is $9 \mathrm{~mm}$. The grill antenna is designed to launch parallel refractive index $\left(N_{/ /}\right)$centered at 2.25 since the elements are relatively phased with adjacent elements at $90^{\circ}\left(\Delta \varphi=90^{\circ}\right)$ when helium gas is used. The machine is operated at a toroidal magnetic field of $1.5 \mathrm{~T}$. Ohmic and vertical field coils are not energized in these experiments.

The power is launched from both the rows in a staggered manner, i.e., initially a $50 \mathrm{~kW}, 20 \mathrm{~ms}$ pulse is launched from one row $\left(\Delta \varphi=0^{\circ}\right.$ phasing $)$ and then immediately after the first pulse, the other row $\left(\Delta \varphi=130^{\circ}\right.$ phasing) launches $50 \mathrm{~kW}$ for next $20 \mathrm{~ms}$. Edge plasma parameters are measured using Langmuir probes installed on both sides of grill antenna. A typical breakdown result is shown in Fig. 2. The top graph shows the ramp voltage and the probe characteristics obtained from Langmuir probe. The measurements reveal formation of dense plasma $\left(n_{\mathrm{e}} \sim 10^{12} \mathrm{~cm}^{-3}\right)$ in the presence of the second LH pulse. The low density plasma formed by the first pulse could not be detected in the Langmuir probe because of its low sensitivity. These two phases of plasma (the low and the high density phase) are also observed in the next plot which shows the spectroscopy results where the total light signal and He6678 line measurements are shown. The
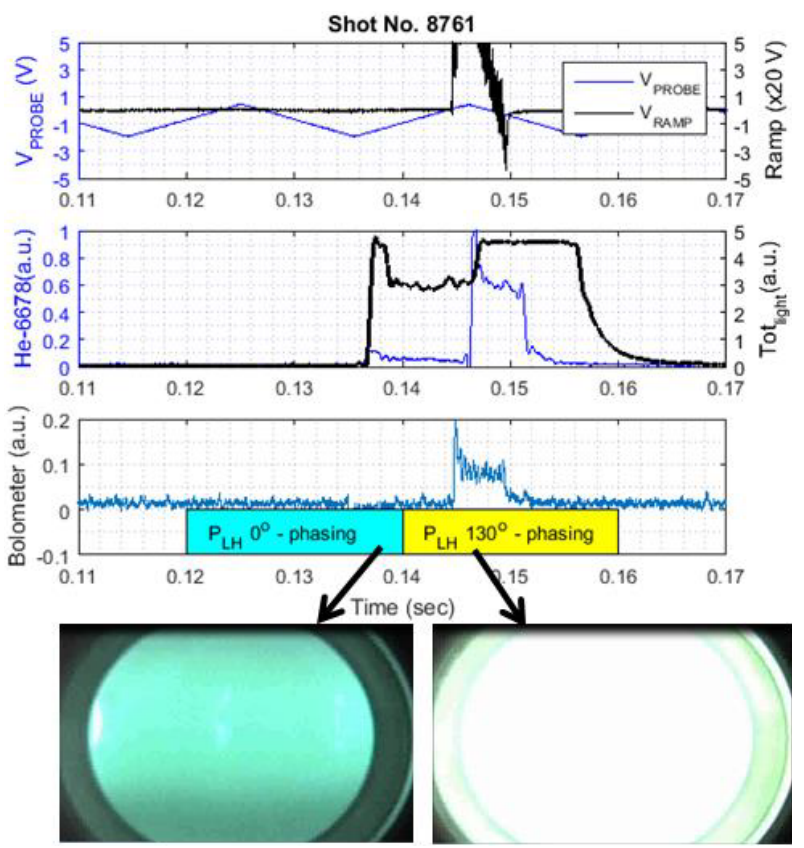

Fig. 2 A typical breakdown result is shown. The top graph shows the results from Langmuir probe whereas the next plot shows the spectroscopy results. The last plot shows the bolometer results along with the LH pulses with different phasing. The two pictures from visible camera viewing the plasma radially, are also shown at the end showing plasma with low density during the first phase and with high density in the second phase.

measurements indicate that after a delay of about $16 \mathrm{~ms}$, the total light signal initially momentarily increases and then settles to a low level. The response of the second pulse is seen after $\sim 5 \mathrm{~ms}$ where the total light signal gets saturated. The same trend is seen in He6678 line. The last plot shows the bolometer results along with the LH pulses with different phasing. The two pictures from visible camera which views the plasma radially, is also shown at the end showing plasma with low density during the first phase and with high density in the second phase. Detailed analysis from the He line indicates formation of low density plasma $\left(n_{\mathrm{e}} \sim 10^{11} \mathrm{~cm}^{-3}\right)$ with the first pulse. In the presence of second pulse of LH power, the density suddenly improved by an order $\left(n_{\mathrm{e}} \sim 10^{12} \mathrm{~cm}^{-3}\right)$ and produced intense plasma. The rise in the density may be attributed to the travelling wave which is launched with finite parallel wavenumber $\left(k_{/ /} \neq 0\right)$. There is small delay in the response of spectroscopy signals when compared with bolometer and probe signals and may be attributed to slow sampling of spectroscopy signals $(1 \mathrm{kHz}$.) compared to higher sampling rate of other signals $(10 \mathrm{kHz}$.)

The layout of the various diagnostics at the SST1 machine is schematically shown in Fig. 3. The Langmuir probe diagnostics is installed near LHCD Grill antenna to measure the edge plasma parameters (low field side of port no. 4). The spectroscopy diagnostics views the plasma from port no. 2. The bolometer diagnostics measures the 


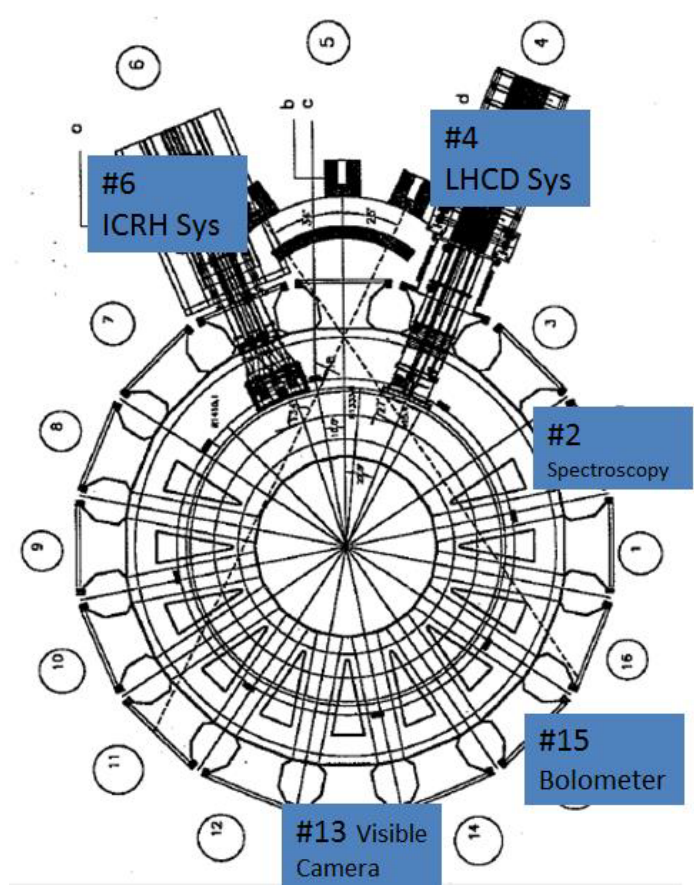

Fig. 3 The layout of diagnostics used for diagnosing the plasma in SST1 machine is shown schematically.

plasma signals from port no. 15. Plasma is detected by visible camera from port no. 13 as well as from port no. 6 where ICRH antenna is installed. These camera view the plasma radially. All these diagnostics further confirmed the formation of plasma in the entire torus of SST1 machine. The plasma could be formed over a range of toroidal magnetic field from $1.2 \mathrm{~T}$ to $1.5 \mathrm{~T}$ and pressure was also varied over $1-5 \times 10^{-4} \mathrm{mbar}$. The pre-ionization of plasma using LHCD system in the absence of loop voltage is observed for the first time in tokamak. Earlier plasma start-up was initiated with LHCD system in PLT (USA) where loop voltage was generated using the poloidal field systems and in JT60U (JAPAN) where a low loop voltage was applied from Ohmic coil system.

Detailed analysis of the spectroscopy signal is shown in Fig. 4. The signals obtained from the He-lines of $667.8 \mathrm{~nm}, 706.5 \mathrm{~nm}$ and $728.1 \mathrm{~nm}$ wavelength is analyzed using Atomic data and analysis structure (ADAS) code [10] to estimate the plasma density and temperature. The density and temperature results obtained from the analysis is shown in the fourth and the fifth plot respectively, which clearly indicates the jump in the density by an order with the travelling waves launched by the second pulse. The temperature in the first phase varies upto $\sim 30 \mathrm{eV}$ and could not be estimated in the presence of second pulse.

The obtained experimental results establishes LH power to be an ideal candidate for forming the preionized target plasma, for plasma startup with low loop voltages necessary for superconducting tokamaks. This ability of LH power would be soon explored in more detail, in SST1 tokamak.

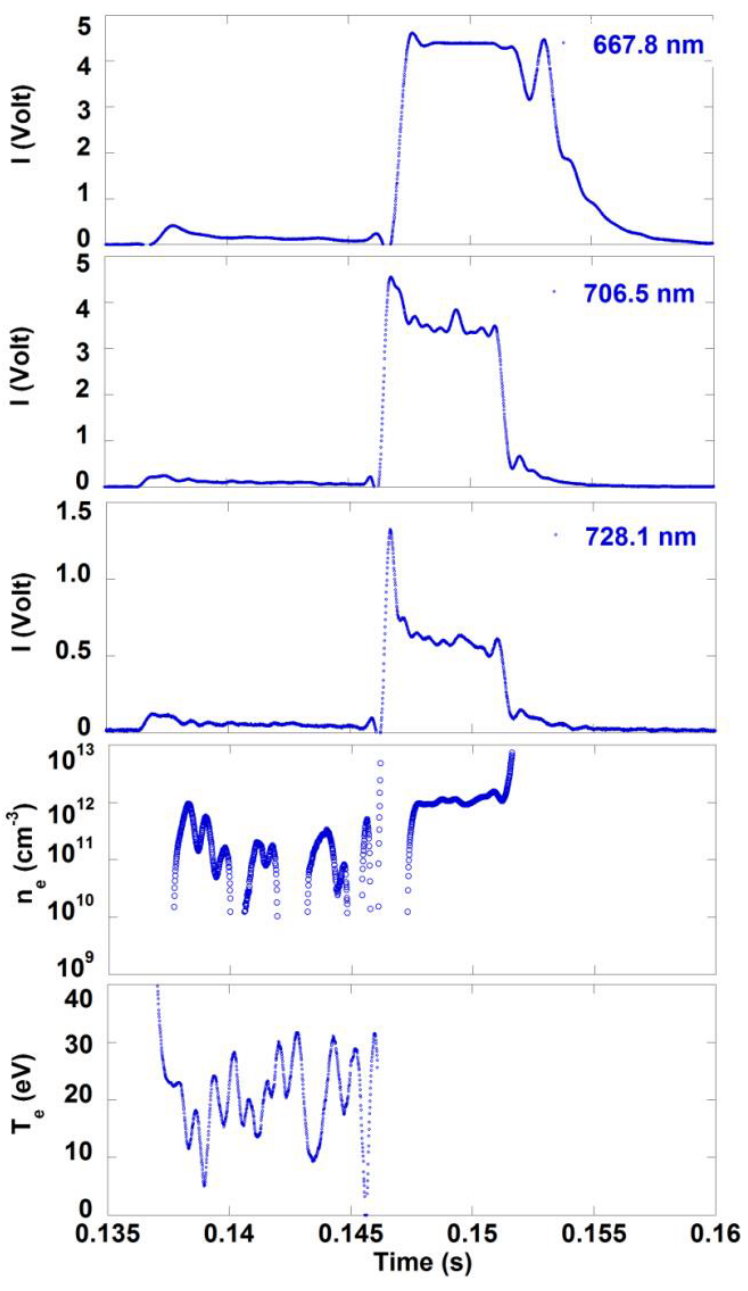

Fig. 4 The evolution of plasma density and temperature using spectroscopy signals and estimated with ADAS code is shown.

\section{Cool Down Experiments on SST1 Machine}

In order to understand the technological issues pertaining to cooling down of the superconducting poloidal field (PF) and toroidal field (TF) coils, for obtaining advanced plasma operation and control, the cooling campaign of both TF and PF system is carried out in a systematic manner. The schematic of different superconducting poloidal coils of SST1 machine is shown in Fig. 5. Initially, the TF coils (16 coils), its casing and PF coils were cooled simultaneously. The temperature (inlet $\sim 15-16 \mathrm{~K}$ and outlet $\sim 24 \mathrm{~K}$ ) for all the coils could be maintained for several hours, but the coils could not be cooled down further. Once this limiting condition was reached, the TF and Case circuits were by passed. Under these conditions, all the PF winding pack attained super-conducting temperature at $5 \mathrm{~K}$. It was established that cooling of PF coils required higher pressure heads which was unexpected and may be attributed to longer path and thus higher hydraulic resistance.

The results obtained from the experiment are shown 


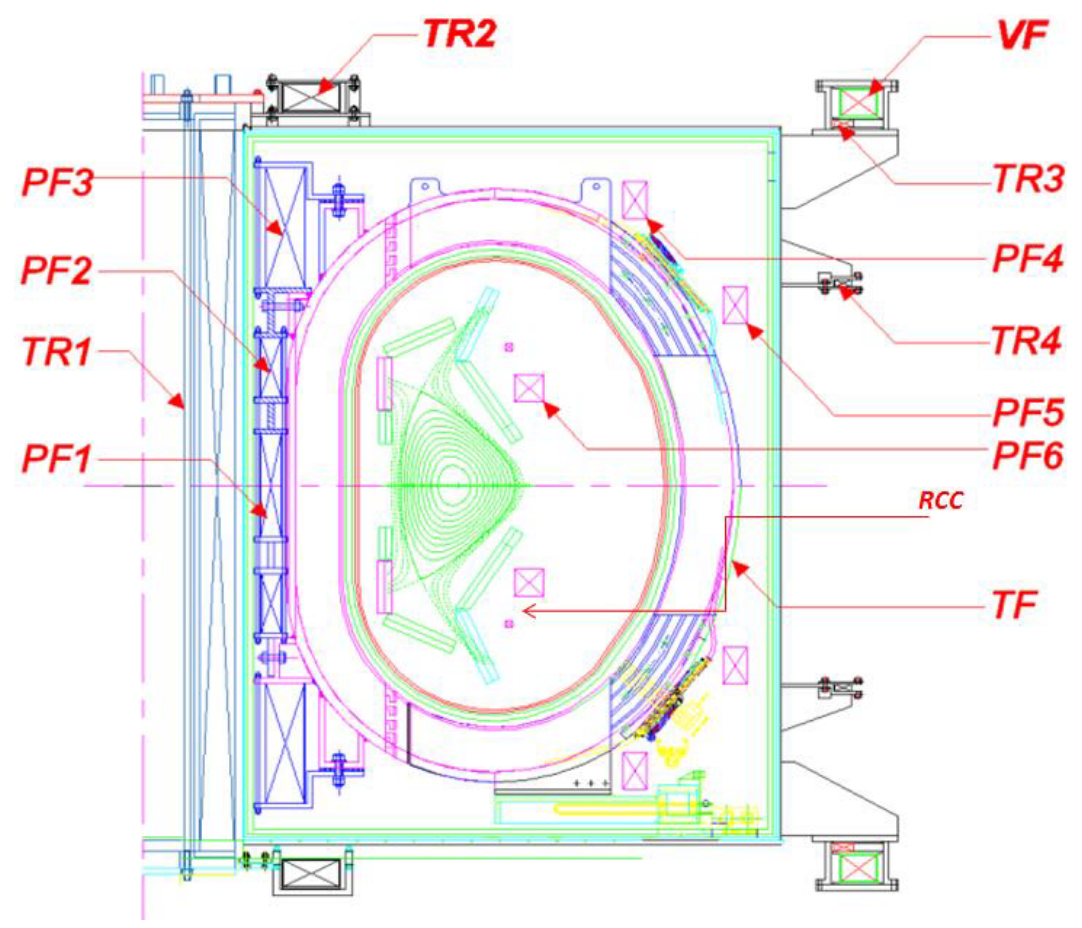

Fig. 5 A schematic of different poloidal field (PF) coils system is shown in poloidal cross section with one toroidal field (TF) coil. The Ohmic transformer (TR) coils, vertical field (VF) coil and radial control coil (RCC) are also shown for reference.

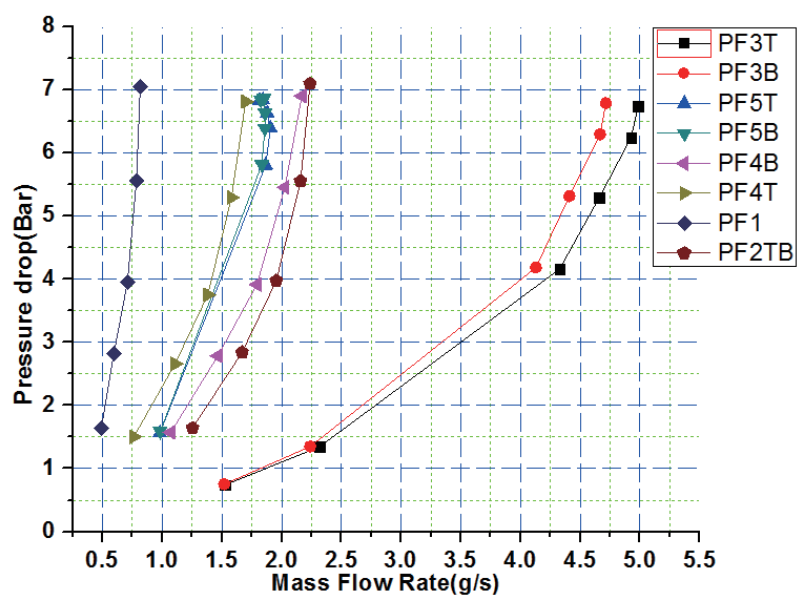

Fig. 6 The curves for PF coils showing mass flow rates with pressure drop across ii is depicted above.

in Fig. 6. Except PF3, all other PF coils have a large gradients and accounts for higher pressure heads reported above. Higher pressure head reflects loss of He-plant capacity. Further additional demand for PF coils would be from current leads, which are not installed at the moment.

\section{Activities on Upgrade of ADITYA Machine}

In SST1 machine, non-inductive current drive employing LHCD was successfully demonstrated. Long discharges assisted with LHCD were repeatedly obtained. The top plot in Fig. 7 shows the Ohmic discharges as- sisted with ECR preionization. However with application of LHCD power, the discharges could be extended beyond $450 \mathrm{~ms}$, which is depicted in the bottom plot in Fig. 7. The coupling issue prevented further current drive temporally and was mainly attributed to poor edge conditions. The coupling studies of LHCD were thus carried out in ADITYA machine [11] using grill antenna [12] to avoid complexities involved in carrying experiments in more demanding superconducting machines.

The experiments were carried out with a single gas puff, injected in to the plasma from the electron flowing side. A typical result obtained is shown in Fig. 8. It is observed that the reflection coefficient decreases with the injection of the gas puff, after a delay of $\sim 25-30 \mathrm{~ms}$. The response of CdTe detector which indicates the generation of supra-thermal electrons with LH power, also shows increase with gas puff, of course after a finite delay and confirms improved coupling and hence strong interaction of LHW's with plasma.

Experiments were carried out to obtain long LHCD discharges by controlling the flow of gas with proper choice of pulse width and pulse gap, thus optimizing the injection of gas puff. It was observed that short $(10 \mathrm{~ms})$ and frequent (every $10 \mathrm{~ms}$ ) gas puff produced better coupling and thus long discharges. Further the current in the Ohmic coils was reduced to lowest level, $\sim 7 \mathrm{kA}$ ( $20 \mathrm{kA}$ gives $0.6 \mathrm{~V}$-s) so that long discharges (beyond $\sim 100 \mathrm{~ms}$ ) cannot be obtained with Ohmic power alone. A typical long discharge obtained with LHCD in the presence of gas puff, lasting for $\sim 210 \mathrm{~ms}$, is shown in Fig. 9. In these ex- 

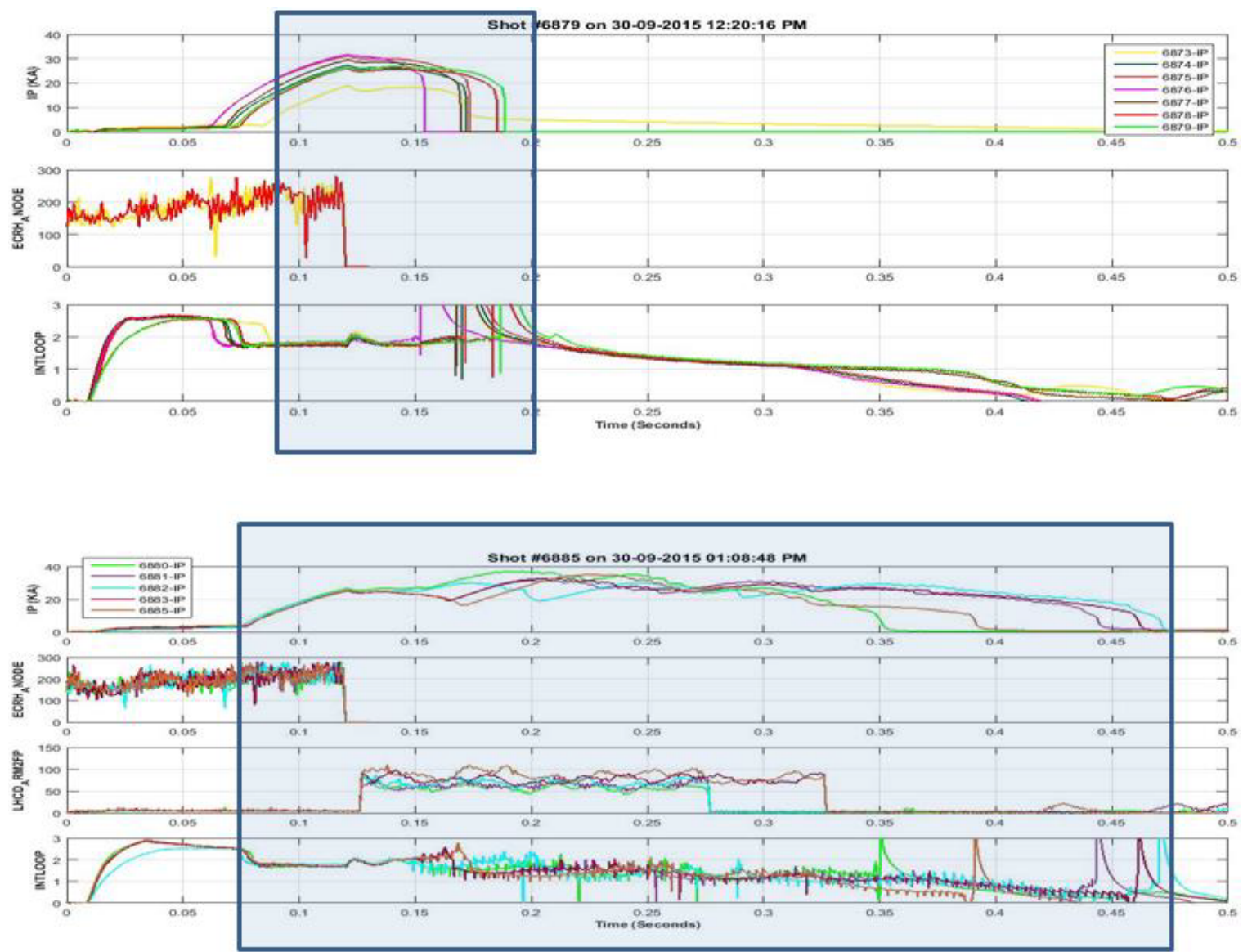

Fig. 7 The plasma shots with non-inductive current drive are compared with purely inductively driven shots. The third and the last plot show the loop voltage (labeled as intloop).

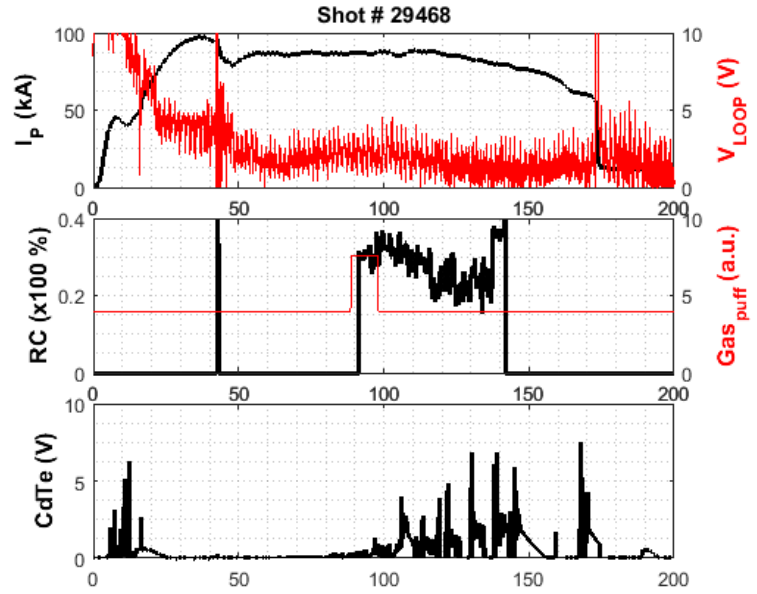

Fig. 8 The improved coupling of LH power with single gas puff is shown. The top plot shows the plasma current and loop voltage. The middle plot shows the pulse during which the gas is puffed from electron flowing side of the antenna. The response of the reflection coefficient is also shown in the same plot. The last plot shows the response of CdTe detector indicating the generation of supra-thermal electrons with LH power.
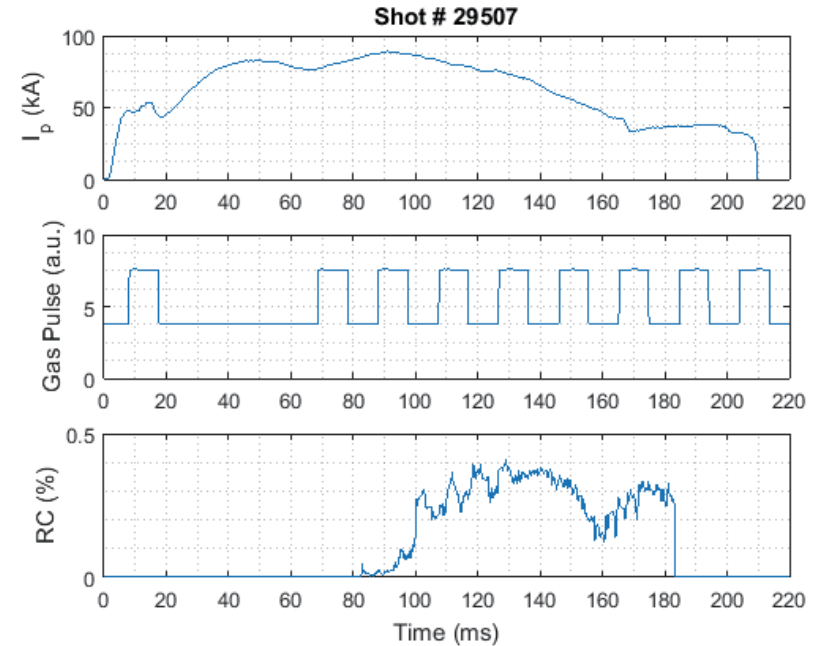

Fig. 9 Long discharges obtained with LHCD employing gas puffing.

periments the reflection coefficient is slightly higher and may be attributed to edge plasma conditions because of reduced current in Ohmic coils.

It has been shown that Passive Active Multijunction (PAM) antenna efficiently couples the LH power to plas- 


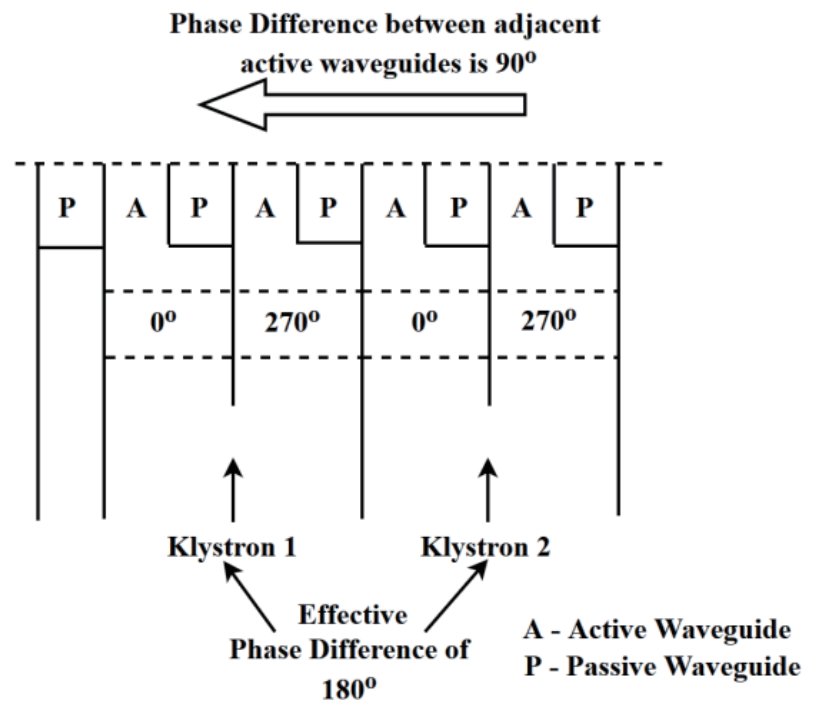

Fig. 10 A schematic of PAM antenna is shown. It shows the top view of the PAM antenna which consists of two modules each powered by separate klystrons. The power in each of the module splits in two parts equally with a phase shift of $270^{\circ}$ in one part employing step phase shifter. It eventually feeds the two active elements (labeled as 'A'). The passive elements (labeled as ' $\mathrm{P}$ ') is a quarter wavelength short, which recirculates the reflected power in to the plasma. Thus there are four active and five passive waveguides in a row.

mas having edge densities close to cut-off $[13,14]$. The PAM antenna thus can be placed away from last closed surface, having poor edge plasma conditions, and still would couple the power very efficiently. Thus a PAM antenna has been designed for ADITYA machine and is under fabrication [15]. It consists of two modules, each having two active and two passive elements, arranged in three rows and its layout is shown in Fig. 10. It consists of two modules each powered by separate klystrons. The power in each of the module splits in to two parts equally with a phase shift of $270^{\circ}$ in one part with the help of step phase shifter. It eventually feeds the two active elements (labeled as 'A'). The passive element (labeled as ' $\mathrm{P}$ ') is a quarter wavelength short, which recirculates the reflected power in to the plasma. Thus there are four active and four passive waveguides in a row. To minimize edge electric effects, an additional passive waveguide is added to the left of first element of first module. To achieve the relative phasing of $270^{\circ}\left(\Delta \varphi=270^{\circ}\right)$, a phase shift of $180^{\circ}$ in klystron-2 would be provided as shown in Fig. 10. The PAM antenna is designed to launch waves with parallel refractive index centered at 2.25 and the launched spectrum is shown in Fig. 11 (top left plot). The reflection coefficient with edge plasma density for the designed PAM antenna is shown in top right plot of Fig. 11. The X-component of electric field in different region of one PAM module is also shown in bottom plot of Fig. 11. The power is fed in $\mathrm{TE}_{10}$ mode from the right. The mode convertor converts it in to $\mathrm{TE}_{30}$ mode

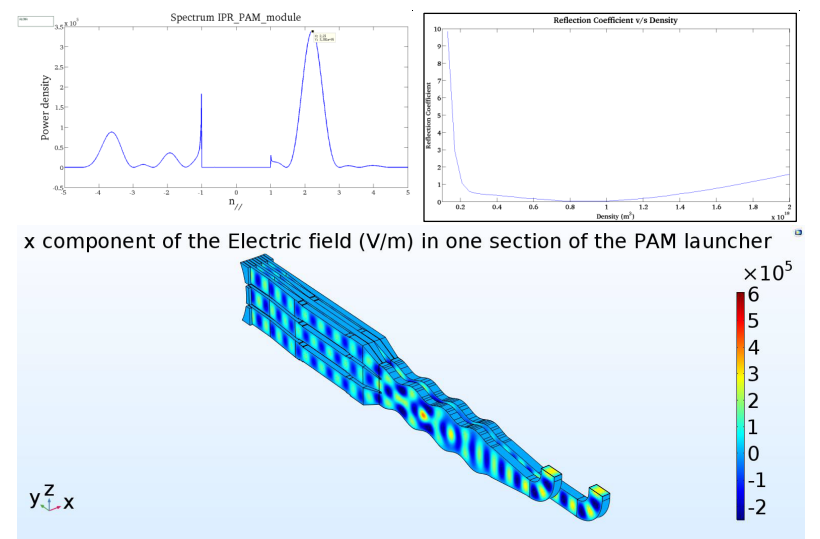

Fig. 11 The PAM antenna is designed to launch waves with parallel refractive index centered at 2.25 and the launched spectrum is shown in top left plot. The top right plot shows the reflection coefficient with edge plasma density. The x-component of the electric field in different region of one PAM module is shown in the bottom plot. The power is fed in $\mathrm{TE}_{10}$ mode from the right. The mode convertor converts it in to $\mathrm{TE}_{30}$ mode which is divided in three layers, to launch $\mathrm{TE}_{10}$ mode from each row.

which is divided in three layers, to launch $\mathrm{TE}_{10}$ mode from each row. Thus new antenna would launch $\sim 300 \mathrm{~kW}$ of $\mathrm{rf}$ power at $3.7 \mathrm{GHz}$ for about a second, as it is not actively cooled.

Due to technical challenges in SST1 machine, it was envisaged to have an alternative where SST1 relevant experiments may be carried out, like testing of PAM concept, divertor operation, etc., with easy access and smaller duty cycle. This would provide insight to technical objectives like double null diverter plasma operation, demonstrate advanced integrated control of plasma, first wall studies with graphite/tungsten wall, etc. and scientific objectives like low loop voltage start up with strong preionization, disruption mitigation studies, runaway mitigations, demonstration of RF heating and current drive schemes, etc. Thus after almost three decades of operation of ADITYA machine, its up-gradation was initiated. Presently the ADITYA-U machine is commissioned and initial experiments are being performed to validate its operation. PAM antenna, which is in advanced stages of fabrication would be installed and commissioned in ADITYA-U for non-inductive current drive experiments.

\section{Conclusions}

In conclusion, it has been demonstrated in SST1, that LHCD has the potential to pre-ionize the gas in the absence of loop voltage and the same may be used for plasma start up with low loop voltage for superconducting tokamaks. Although some technical issues persists with the cool down of Cases, PF and TF coils simultaneously, but SST1 relevant experiments may be carried out in ADITYA-U machine which has been installed and success- 
fully commissioned. New system like PAM antenna which is in advanced stage of fabrication would be installed in ADITYA-U soon and will provide solution for coupling issues leading to long duration discharges produced with non-inductive LH current drive method.

[1] D. Van Houtte and Equipe TORE SUPRA, Nucl. Fusion 33, 137 (1993).

[2] Y.C. Saxena and SST1 Team, Nucl. Fusion 40(6), 1069 (2000).

[3] B. Wan and International Collaborators, Nucl. Fusion 40, 1057 (2000).

[4] H.L. Yang et al., Fusion Eng. Des. 86, 588 (2011).

[5] F.W. Perkins, D.E. Post, N.A. Uckan, M. Azumi et al., Nucl. Fusion 39, 2137 (1999).

[6] D. Bora, P.K. Sharma, S.L. Rao, R.G. Trivedi et al., Fusion Eng. Des. 82, 141 (2007).
[7] P.K. Sharma, Fusion Sci. Technol. 65, 103 (2014).

[8] F. Jobes, J. Stevens, R. Bell, S. Bernabei et al., Phy. Rev. Lett. 52(12), 1005 (1984).

[9] M. Seki and R. Yoshino, Plasma Phys. Control. Fusion 39, 205 (1997).

[10] H.P. Summers, "Atomic data and analysis structure", JET, Report No. JET-IR(94)-06 (1994).

[11] S.B. Bhatt, D. Bora, B.N. Buch, C.N. Gupta et al., Indian J. Pure Appl. Phys. 27, 710 (1989).

[12] P.K. Sharma, S.L. Rao, D. Bora, R.G. Trivedi et al., Fusion Eng. Des. 82, 41 (2007).

[13] F. Mirizzi, Ph. Bibet, A.A. Tuccillo et al., Proc. of the 22nd SOFT, 9-13 Sept., Helsinki, Finland (2002).

[14] A. Ekedahl, L. Delpech, M. Goniche, D. Guilhem et al., Nucl. Fusion 50, 112002 (2010).

[15] Y.M. Jain and P.K. Sharma, 32nd National Symposium on Plasma Science and Technology (Plasma-2017), 07-10, Nov., Gandhinagar, India (2017). 